\title{
Les peuplements de mollusques de la zone littorale du lac d'Annecy (Savoie, France)
}

\author{
J. Mouthon 1 \\ J.P. Dubois ${ }^{2}$
}

Mots-clés : mollusques, lac, évolution des peuplements.

A l'occasion de recherches sur la dermatite cercarienne qui affecte le lac d'Annecy, un des plus grands lacs alpins français, un inventaire des populations de mollusques de sa zone littorale a été réalisé. Les gastéropodes sont assez mal représentés et les espèces qui sont généralement des éléments dominants de la faune lacustre comme Valvata piscinalis, Bithynia tentaculata ou Planorbis carinatus sont peu abondantes. Les nombreux aménagements du littoral qui ont fortement réduit la superficie des ceintures végétales sont probablement responsables de cette situation. En revanche, les peuplements de Sphaeriidae, dominés par Pisidium subtruncatum, $P$. henslowanum et $P$. moitessierianum sont abondants et bien diversifiés.

Au cours des 40 dernières années, 6 nouvelles espèces se sont installées dans le lac d'Annecy. Trois d'entre elles ont actuellement largement colonisé le plan d'eau. Il s'agit de Dreissena polymorpha, Potamopyrgus antipodarum et de Pisidium moitessierianum. L'apparition récente de cette dernière, qui vit seulement dans les grands lacs de plaine et le potamon des cours d'eau contrairement aux autres Pisidium du lac, pourrait être une conséquence du réchauffement climatique.

\section{Mollusc communities of the littoral zone of Annecy Lake (Savoie, France)}

Keywords : molluscs, lake, community change.

Using the opportunity of research on cercarial dermatitis which is of concern in lake Annecy, one of the largest alpine lakes of France, an inventory of mollusc communities was carried out. Gastropods and particularly dominant species of lacustrine lakes like Valvata piscinalis, Bithynia tentaculata or Planorbis carinatus are not very abundant. The many littoral developments which have strongly reduced the vegetation belt area are probably responsible for this situation. On the other hand, sphaeriids dominated by Pisidium subtruncatum, $P$. henslowanum and $P$. moitessierianum are abundant and well diversified.

During the forty last years, six new species colonized L. Annecy. At present, three of these (Dreissena polymorpha, Pisidium moitessierianum and Potamopyrgus antipodarum) are abundant in the littoral zone. The recent arrival of $P$. moitessierianum, which only lives in large plane lakes and in the potamon of rivers, unlike the others Pisidium of Annecy, could be a result of climatic change.

\section{Introduction}

«Encerclé de villas et d'hôtels dont les eaux usées vont droit au lac dont presque tout le pourtour a été modifié». C'est en ces termes qu'Hubault décrit en

\footnotetext{
1. Cemagref, 3 bis quai Chauveau, CP 220, F-69336 Lyon cedex 09. E-mail : jacques.mouthon@cemagref.fr

2. INRA, Station d'hydrobiologie lacustre, 75 avenue de Corzent, B.P. 511. Thonon-les-Bains.

E-mail : dubois@thonon.inra.fr
}

1947 l'emprise de l'homme sur le lac d'Annecy. Cette pollution devenant de plus en plus préoccupante et préjudiciable aux activités touristiques, la décision de construction d'un collecteur des eaux usées a été prise en 1957. Après la réalisation de ces importants travaux d'assainissement qui s'est achevée en 1998, il était intéressant de procéder à un inventaire des peuplements de mollusques de ce lac.

En effet, si pour le Léman (essentiellement le Petit Lac ou lac de Genève) et le Bourget des relevés malacologiques détaillés on été effectués dans le passé (Favre, $1927 ; 1940$ ), il ressort des recherches biblio- 
graphiques que nous ne disposons que de bien peu de données sur les peuplements de mollusques du lac d'Annecy, un des plus grands lacs naturels français. Les premières récoltes ont été effectuées lors de recherches biologiques de 1895 à 1905 (Le Roux 19071908). Toutefois, la systématique ayant considérablement évolué depuis cette époque la liste faunistique proposée est peu utilisable. Une vingtaine de dragages ont été réalisés par Favre (1940), sans doute entre les années 1929 et 1939 , la date exacte n'étant pas connue, dans la partie nord du lac à différentes profondeurs. Cette collection, déposée au Muséum d'Histoire naturelle de Genève, n'ayant jamais fait l'objet de publication a été étudiée dans le cadre de ce travail et a permis de montrer l'évolution des peuplements littoraux de cette partie du lac.

Depuis quelques années, on constate une recrudescence de la dermatite cercarienne et des bilharzies aviaires dans les grands lacs savoyards, comme dans de nombreux plans d'eau de France (Gentile et al, 1996, Martins 2000). C'est à l'occasion de recherches effectuées sur les populations de mollusques et plus particulièrement des gastéropodes appartenant à la famille des Lymnaeidae, principaux hôtes intermédiaires de ces parasites Trématodes dans le lac d'Annecy, que fut réalisé l'inventaire qui fait l'objet de ce travail.

\section{Matériel et méthodes}

Les mollusques des habitats sédimentaires de la zone littorale du lac d'Annecy (alt. 446,5 m, long. $14 \mathrm{~km}$, larg. $5 \mathrm{~km}$, prof. $64,7 \mathrm{~m}$ ) ont été récoltés : - entre 0 et : 1,5-2 m, à l'aide d'un troubleau à base rectangulaire $(25 \times 18 \mathrm{~cm})$, vide de maille $630 \mu \mathrm{m}$, une surface de 1 à $2 \mathrm{~m}^{2}$ étant échantillonnée suivant les sites (Stations 1 à 19) ; - entre -2 et $-3 \mathrm{~m}$, à l'aide d'une benne Friedinger $\left(S=350 \mathrm{~cm}^{2}\right)$, cinq prélèvements réunis dans un seul échantillon étant réalisés sur chaque site (Stations 20 à 27). Les échantillons furent tamisés à $630 \mu \mathrm{m}$, puis triés et déterminés à l'aide d'une loupe binoculaire. Les densités des espèces, sur chaque station, ont ensuite été rapportées au $\mathrm{m}^{2}$. Les fréquences des espèces ont été obtenues en divisant le nombre de stations sur lequel l'espèce a été récoltée par le nombre total de stations échantillonnées : 19 entre 0 et $-2 \mathrm{~m}$, 8 entre -2 et $-3 \mathrm{~m}$. Les prélèvements ont été réalisés au cours des deux premières semaines de Juin 2000 . Les dragages effectués par Favre dans la zone littorale de la partie nord du lac ont été réalisés dans le secteur compris entre les stations 14 et 19.

\section{Résultats}

Au total, 14 espèces de gastéropodes et 16 espèces de bivalves ont été répertoriées dans le lac d' Annecy (Annexe 1).

\subsection{De 0 à $-2 \mathbf{m}$ de profondeur}

Les gastéropodes sont représentés par 13 espèces. Si l'on excepte Potamopyrgus antipodarum dont la fréquence est supérieure à $50 \%$, seules quatre espèces : Bithynia tentaculata, Physella acuta, Valvata piscinalis et $V$. cristata sont présentes sur plus de $25 \%$ des stations (Tableau 1). Avec une densité moyenne de 230 ind $/ \mathrm{m}^{2}, P$. antipodarum constitue l'essentiel des effectifs des gastéropodes sur la plupart des stations ; les densités moyennes par mètre carré des autres espèces demeurant inférieures à $6 \mathrm{ind} / \mathrm{m}^{2}$.

Contrairement aux gastéropodes, la plupart des 16 espèces de bivalves recensées sont bien représentées sur l'ensemble des stations. En effet, 8 espèces atteignent des fréquences supérieures ou égales à $50 \%$ alors que seulement trois d'entre elles Unio pictorum, Sphaerium corneum et Pisidium personatum, sont présentes dans moins de $25 \%$ des stations. Avec des densités moyennes supérieures à $50 \mathrm{ind} / \mathrm{m}^{2}$, Pisidium $s u b$ truncatum, $P$. moitessierianum, $P$. henslowanum et $P$. casertanum sont les bivalves les mieux représentés dans la zone littorale du lac d'Annecy ; des densités supérieures à $500 \mathrm{ind} / \mathrm{m}^{2}$ ayant même été relevées pour $P$. subtruncatum et $P$. moitessierianum. Toutefois, d'autres espèces peuvent être localement abondantes puisque 7 Pisidium atteignent des abondances maximales dépassant $100 \mathrm{ind} / \mathrm{m}^{2}$. Parmi les espèces peu représentées dans les habitats littoraux sédimentaires figurent $S$. corneum, $P$. personatum, Musculium lacustre et Dreissena polymorpha. Toutefois, les nombreux enrochements mis en place lors de l'aménagement du plan d'eau constituant l'habitat préférentiel de $D$. polymorpha, ce bivalve est aujourd'hui largement répandu dans la zone littorale du lac mais ce type d'habitat n'a pas fait l'objet d'un échantillonnage quantitatif.

Sur la figure 1, on observe l'évolution de l'amont (Bout du Lac) vers l'aval (Albigny) de la densité par mètre carré et du nombre d'espèces de gastéropodes et de bivalves. Le plus souvent, les abondances des gastéropodes sont faibles et c'est seulement au niveau de Duingt, St-Jorioz (St. 4 à 8) et Albigny (St. 17, 18, 19), où $P$. antipodarum est généralement bien représenté, que les effectifs atteignent des valeurs plus élevées. En revanche, les bivalves présentent de fortes abondances sur toutes les stations prospectées, sauf au niveau du point 4 (Duingt), situé à proximité de l'arrivée du ruisseau d'Entrevernes. 


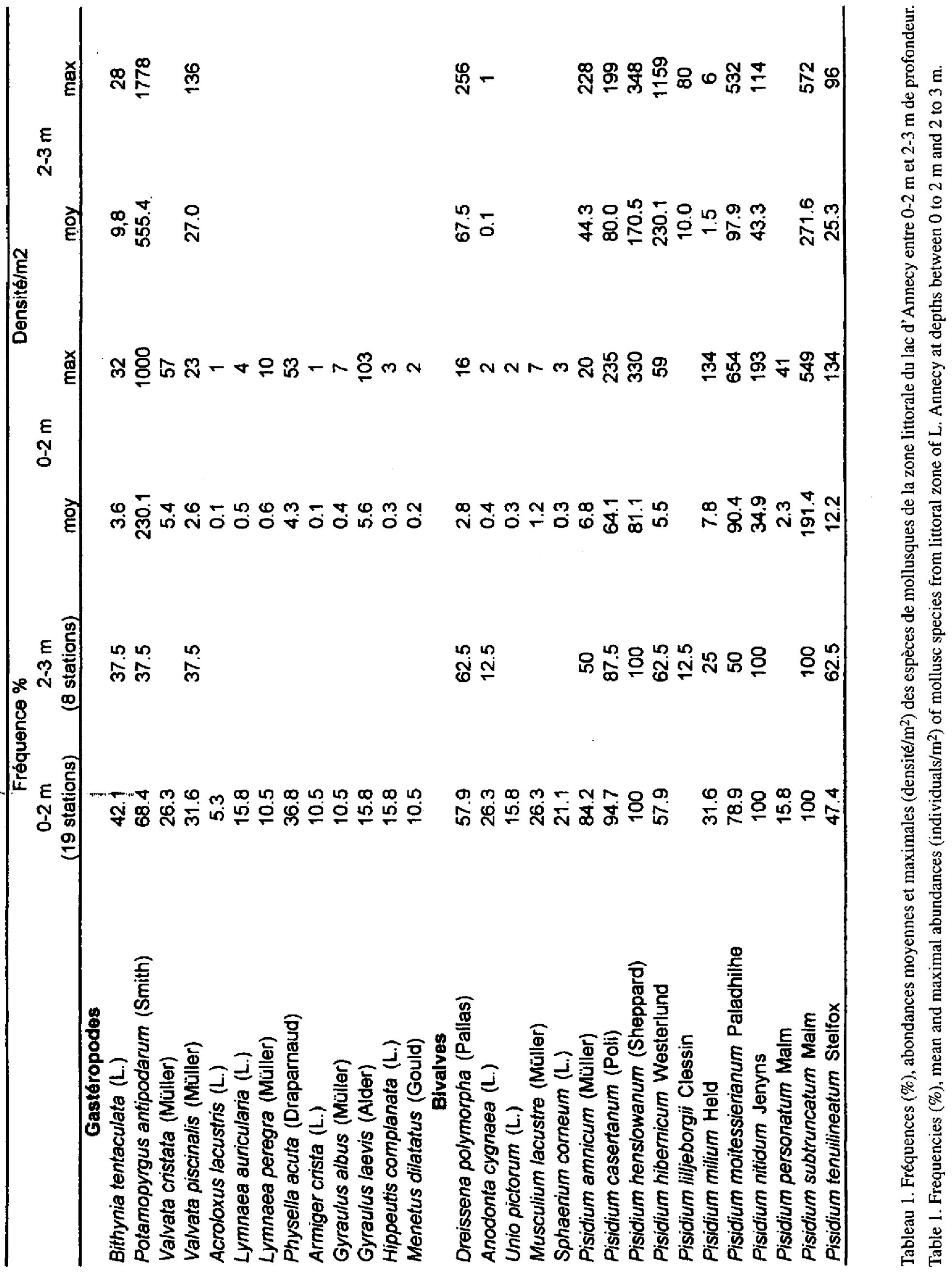




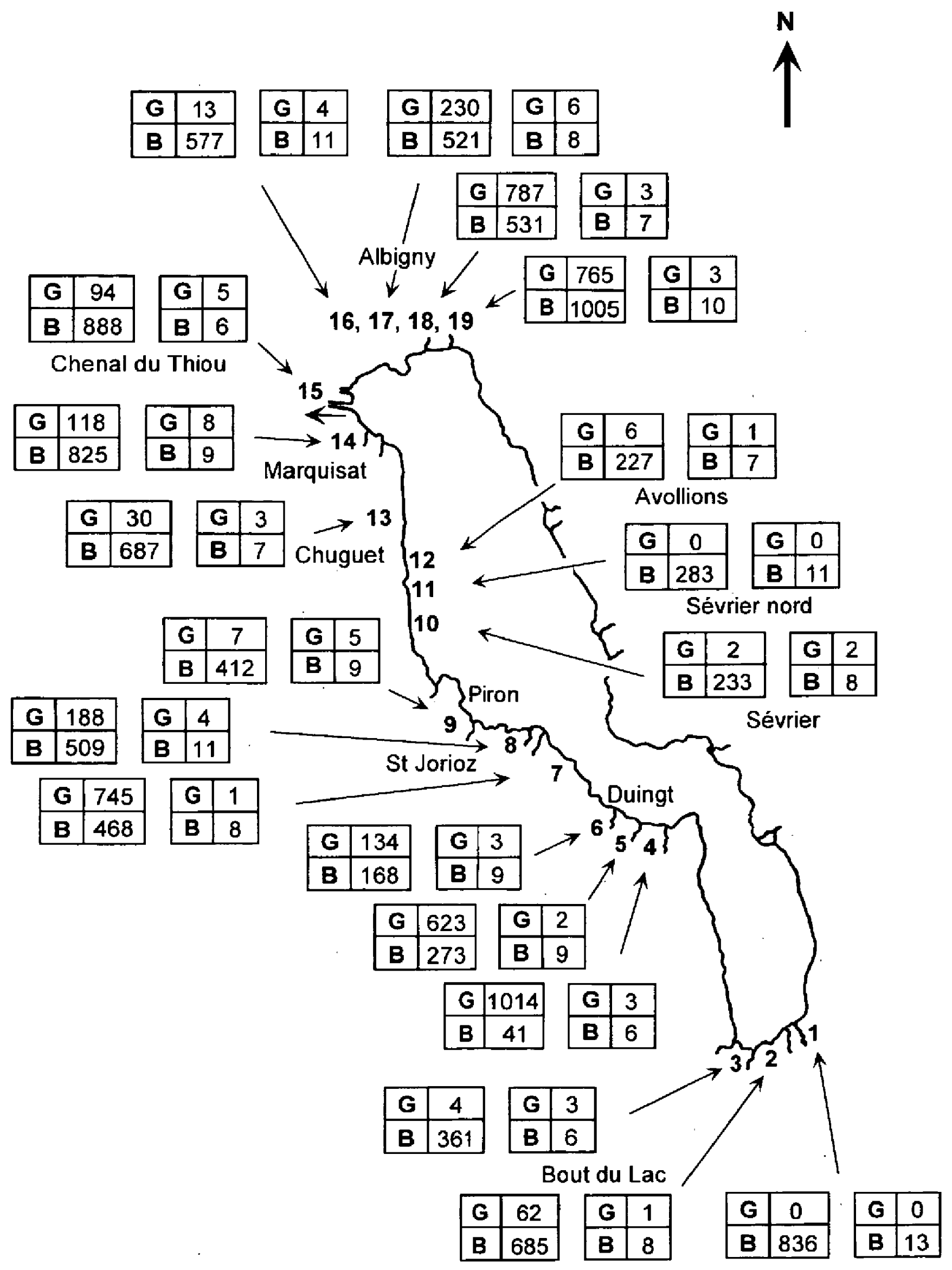

Fig. 1. Effectifs (premier rectangle) et richesse spécifique (deuxième rectangle) des gastéropodes (G) et des bivalves (B) de 19 stations (numéros en gras) du lac d'Annecy entre 0-2 $\mathrm{m}$ de profondeur.

Fig. 1. Abundances (first rectangle) and species richness (second rectangle) of gastropods (G) and bivalves (B) from 19 stations (numbers in bold) of L. Annecy at depths between 0 and $2 \mathrm{~m}$. 
La richesse spécifique des gastéropodes est le plus souvent modeste, seules 4 stations hébergent au moins 5 espèces. Outre Piron (9) où les densités relevées sont par ailleurs très faibles, c'est essentiellement dans la partie nord du lac d'Annecy : stations 17 (Albigny), 15 (chenal du Thiou) et surtout 14 (Les Marquisats) que les peuplements de gastéropodes sont les plus diversifiés. Si $P$. antipodarum, largement distribué dans la zone littorale du lac atteint son abondance maximale à Duingt (4), c'est bien dans la partie nord du plan d'eau que Gyraulus laevis, P. acuta, V. piscinalis, B. tentaculata et $V$. cristata sont les mieux représentés (Annexe 1). A ces espèces, il faut ajouter Lymnaea peregra (forme ovata) qui prolifère dans le secteur caillouteux non échantillonné situé immédiatement à l'ouest de la plage d'Albigny, parfois en compagnie de $L$. auricularia dont la densité demeure cependant beaucoup plus modeste (Hubert 2000). Lors d'une prospection des habitats caillouteux des rives du lac réalisée dans le cadre des recherches sur la dermatite cercarienne, $L$. auricularia a également été découverte au niveau des ports d'Albigny et d'Annecy, des Marquisats, de Piron, de St-Jorioz, des plages d'Angon, Talloires et de Menthon, et $L$. peregra (forme ovata), à Piron et à proximité de l'embouchure de plusieurs ruisseaux qui se jettent dans le lac.

Les peuplements de bivalves du littoral du lac, à l'exception du chenal du Thiou (station 15) sont dominés par trois espèces : $P$. subtruncatum (11 St.), $P$. henslowanum (4 St.) et $P$. moitessierianum (3 St.) (figure 2). La structure de ces peuplements se caractérise par la forte représentation d'une seule espèce pour 8 des 18 stations. En revanche, les autres stations hébergent des peuplements plus équilibrés où plusieurs espèces atteignent des abondances voisines. C'est notamment le cas de la station 9 (Piron) où les abondances relatives de 5 Pisidium sont comprises entre 12 et $23,5 \%$. Peu abondante dans les peuplements littoraux, Pisidium tenuilineatum atteint sa plus forte densité (134 ind $/ \mathrm{m}^{2}$ ) au niveau de la station 7 (St-Jorioz). $P$. personatum, commun dans la zone profonde des lacs, colonise préférentiellement les eaux souterraines et les sources (Favre 1927, 1940, Kuiper 1966, MeierBrook 1975). Sa présence au niveau des stations 1, 2 (Bout du Lac) et surtout 6 (Duingt) est liée à l'arrivée de ruisseaux comme celui d'Entrevernes. Cinq espèces $D$. polymorpha, $M$. lacustre, S. corneum, Anodonta cygnea et Unio pictorum présentant des densités très faibles ont été regroupées dans "autres bivalves" (Annexe 1). Les variations que l'on observe dans la structure des peuplements de bivalves de la zone littorale du lac d'Annecy entre 0 et $2 \mathrm{~m}$ traduisent une forte hété- rogénéité des habitats sédimentaires et des conditions qui y règnent.

Dans le chenal du Thiou (St. 15), les peuplements de bivalves sont essentiellement représentés par trois espèces $P$. subtruncatum $(50,6 \%), P$. casertanum (26,5\%) et $P$. nitidum $(21,7 \%$ ) (Annexe 1$)$. On retrouve à ce niveau une association d'espèces caractéristiques de cours d'eau appartenant au rhithron (Mouthon 1981, 1999), où seule la forte représentation de $P$. casertanum rappelle l'influence lacustre.

\subsection{De - 2 à $\mathbf{- 3} \mathrm{m}$ de profondeur}

Entre 2 et 3 mètres, seules trois espèces de gastéropodes sont recensées dans les 8 stations : Bithynia tentaculata, $P$. antipodarum et V. piscinalis (Annexe 1) ; elles présentent la même fréquence $(37,5 \%)$. Toutefois, $P$. antipodarum atteint des abondances moyennes élevées $\left(555,4 \mathrm{ind} / \mathrm{m}^{2}\right)$, alors que celles des deux autres espèces demeurent relativement faibles, respectivement 9,8 et $27 \mathrm{ind} / \mathrm{m}^{2}$ (Tableau 1). Comme dans la bande littorale comprise entre 0 et $2 \mathrm{~m}$, les bivalves sont ici plus largement représentés que les gastéropodes. En effet, 9 espèces sur les 12 recensées atteignent des fréquences égales ou supérieures à $50 \%$, et trois Pisidium $P$. henslowanum, $P$. nitidum et $P$. subtruncatum sont présents sur les 8 stations échantillonnées. Les densités moyennes relevées atteignent 271,6 ind $/ \mathrm{m}^{2}$ pour l'espèce la mieux représentée $P$. subtruncatum, et respectivement 230,1 et $170,5 \mathrm{ind} / \mathrm{m}^{2}$ pour $P$. hibernicum et $P$. henslowanum ; elles sont inférieures à $100 \mathrm{ind} / \mathrm{m}^{2}$ pour les autres $P$ isidium.

L'espèce dominante varie d'un secteur à l'autre du lac (Fig. 3). Du sud au nord, elle est successivement représentée par $P$. subtruncatum (St. 20,21,22), au niveau de Piron (St. 23, 24) par P. hibernicum, dans le secteur de Sévrier (St. 25, 26) par P. henslowanum et à Albigny (St. 27) par $P$. moitessierianum. L'hétérogénéité des conditions abiotiques et biotiques entre 2 et $3 \mathrm{~m}$ apparaît donc encore plus élevée que dans la bande de profondeur comprise entre 0 et $2 \mathrm{~m}$. La baie de Piron - Les Mongets, par la largeur de son plateau littoral, semble constituer une zone refuge pour $P$ isidium lilljeborgii qui n'a été découvert que sur ce site. C'est également dans ce secteur du lac que $P$. hibernicum, espèce très commune dans les milieux lacustres (Kuiper 1966, Meier-Brook 1975, Mouthon \& Kuiper 1987), atteint son abondance maximale (1159 ind $\left./ \mathrm{m}^{2}\right)$.

\section{Discussion}

D'après Le Roux (1907-1908) et l'étude de la collection de Favre de la partie Nord du lac, 6 nouvelles 

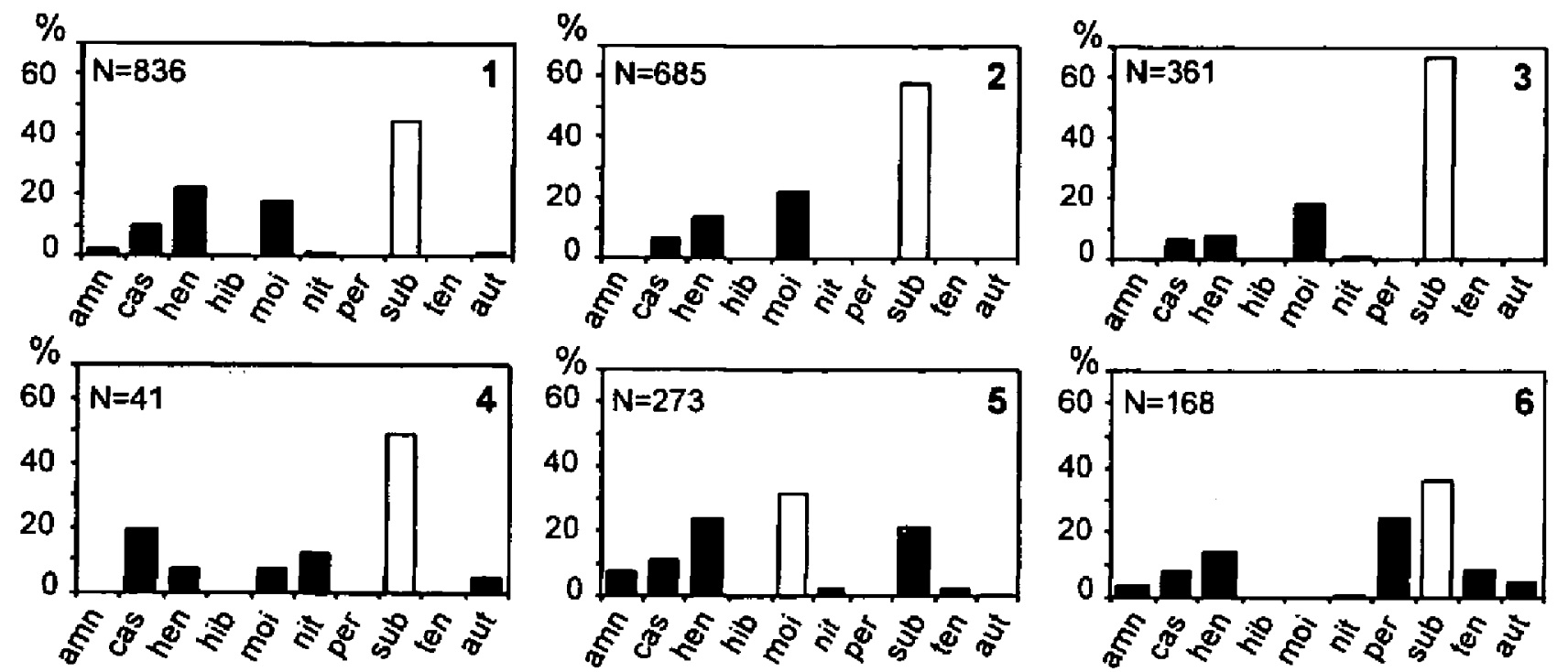

ริ
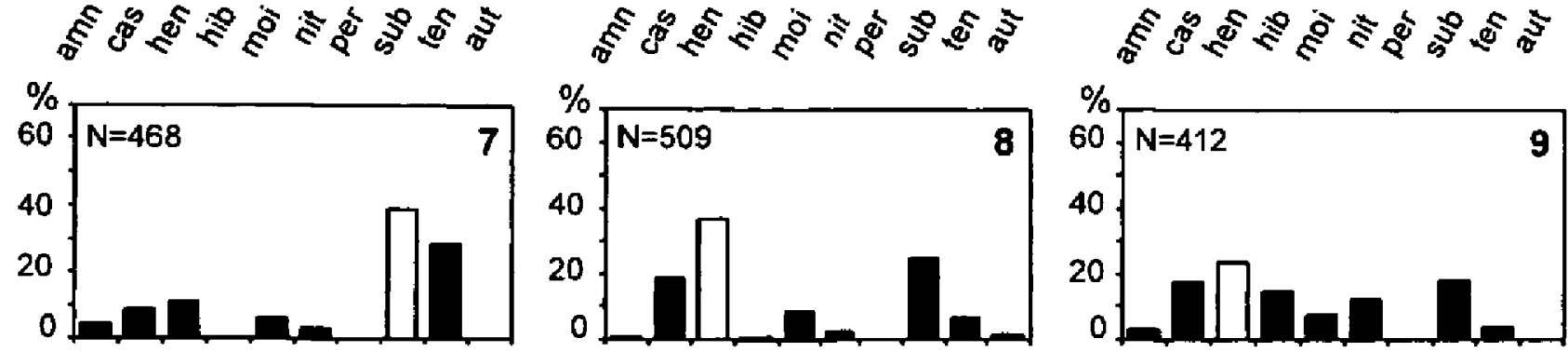

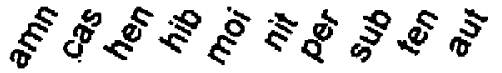
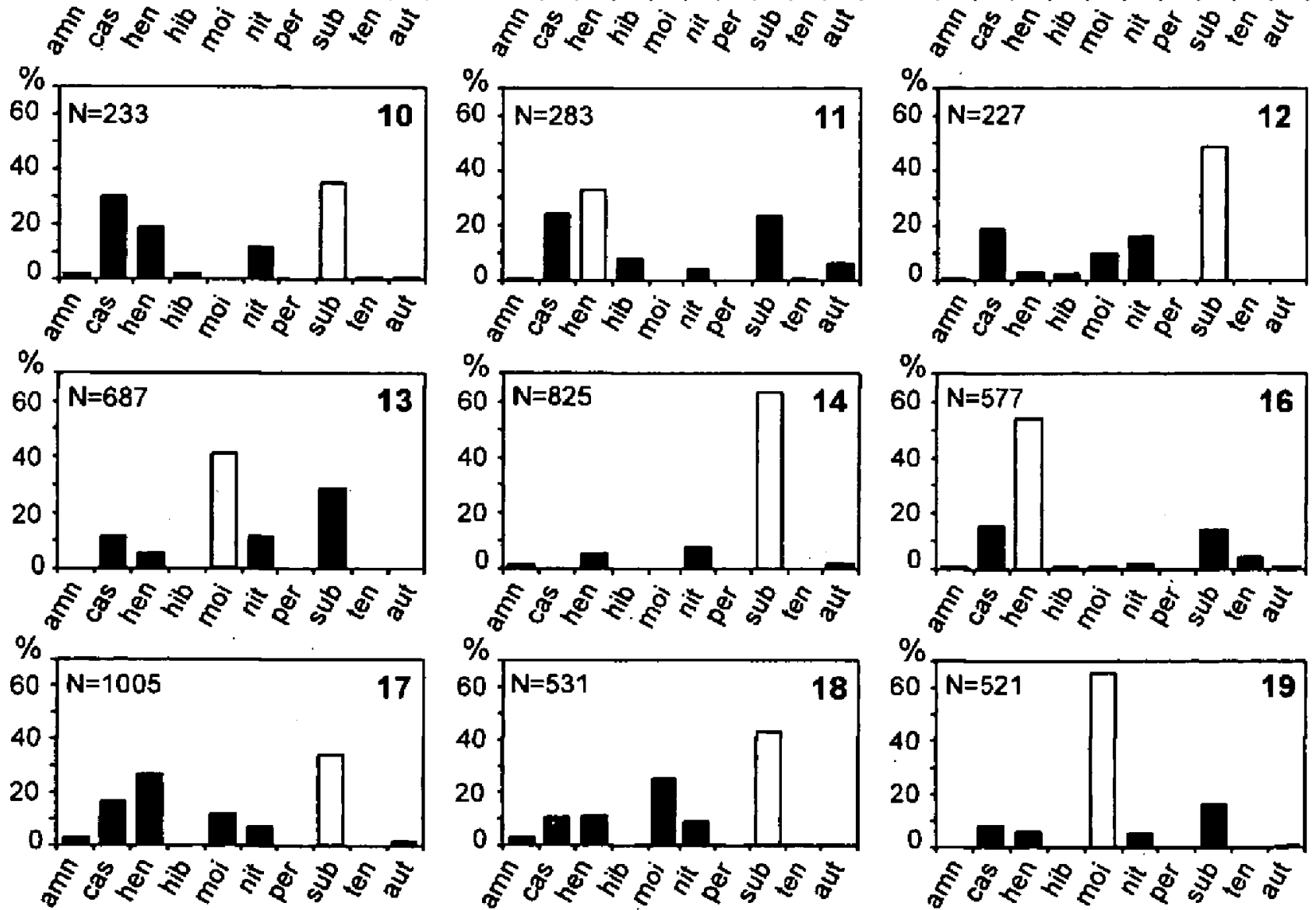

Fig. 2. Abondances relatives des espèces de bivalves présentes sur 18 stations (numéros en gras) du lac d'Annecy entre 0 et $2 \mathrm{~m}$ de profondeur ( $\mathrm{N}=$ abondance totale $/ \mathrm{m}^{2}$, l'histogramme de l'espèce dominante est en blanc) (amn $=$ Pisidium amnicum, cas $=P$. casertanum, hen $=P$. henslowanum, hib $=P$. hibernicum, moi $=P$. moitessierianum, nit $=P$. nitidum, per $=P$. personatum, sub $=P$. subtruncatum, ten $=P$. tenuilineatum, aut $=$ autres espèces de bivalves - $D$. polymorpha, $M$. lacustre, $S$. corneum, A. cygnaea et $U$. pictorum).

Fig. 2. Relative abundances of bivalve species sampled from 18 stations (numbers in bold) of L. Annecy at depths from 0 to $2 \mathrm{~m}$. ( $\mathrm{N}=$ individuals $/ \mathrm{m}^{2}$, histogramm of dominant species is in white) (amn = Pisidium amnicum, cas $=P$. casertanum, hen $=P$. henslowanum, hib $=P$. hibernicum, moi $=P$. moitessierianum, nit $=P$. nitidum, per $=P$. personatum, sub $=P$. subtruncatum, ten $=P$. tenuilineatum, aut $=$ other bivalve species $-D$. polymorpha, M. lacustre, $S$. corneum, A. cygnaea and $U$. pictorum). 


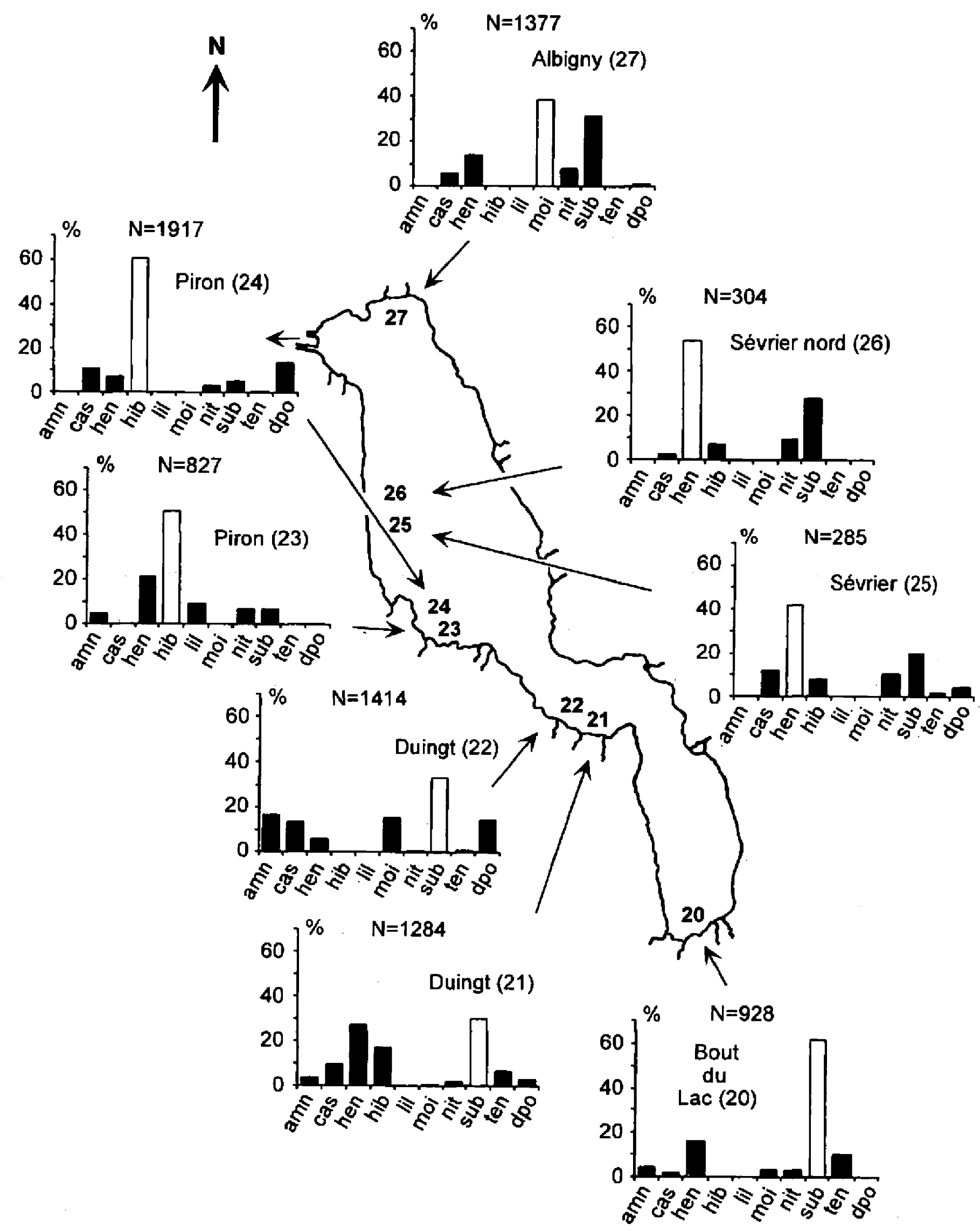

Fig. 3. Abondances relatives des espèces de bivalves présentes sur 8 stations (numéros en gras) du lac d'Annecy entre 2 et $3 \mathrm{~m}$ de profondeur ( $\mathrm{N}=$ abondance totale $/ \mathrm{m}^{2}$, l'histogramme de l'espèce dominante est en blanc) (amn $=$ Pisidium amnicum, cas $=P$. casertanum, hen $=P$. henslowanum, hib $=P$. hibernicum, lil $=P$. lilljeborgii, moi $=$ $P$. moitessierianum, nit $=P$. nitidum, sub $=P$. subtruncatum, ten $=P$. tenuilineatum, dpo $=D$. polymorpha) .

Fig. 3. Relative abundances of bivalve species sampled from 8 stations (numbers in bold) of Annecy lake at depths from 2 to $3 \mathrm{~m}$. (N = individuals $/ \mathrm{m}^{2}$, histogramm of dominant species is in white) (amn = Pisidium amnicum, cas $=P$. casertanum, hen $=P$. henslowanum, hib $=P$. hibernicum, lil $=P$. lilljeborgii, moi $=P$. moitessierianum, nit $=$ $P$. nitidum, sub $=P$. subtruncatum, ten $=P$. tenuilineatum, $\mathrm{dpo}=D$. polymorpha . 
espèces de mollusques sont décrites dans ce plan d'eau. La première à avoir colonisé le lac est sans doute Dreissena polymorpha. Originaire de la région Ponto-Caspienne, ce bivalve est, d'après Juget (1967), apparu simultanément dans le Léman où sa présence a été signalée en 1962 (Binder 1965) et à Annecy. La seconde $P$. antipodarum est originaire de Nouvelle-Zélande. Elle n'est pas mentionnée en Savoie fin 1972, lors de l'établissement de la carte de répartition de ce gastéropode par Real (1974), mais elle est signalée dans le Léman (Petit-Lac) en 1977 (Crozet et al., 1980) ; son installation à Annecy date probablement de la même période. Quatre autres espèces sont sans doute apparues plus récemment dans le Nord du lac d'Annecy. Il s'agit des Planorbidae Gyraulus laevis et Menetus dilatatus, de P. acuta (Physidae) et de P. moitessierianum (Sphaeriidae).

Menetus dilatatus, présente uniquement dans le secteur de Duingt, est originaire d'Amérique du Nord et a récemment envahi la France via les Iles Britanniques (Mouthon 1986). P. acuta et G. laevis sont surtout bien représentées dans le port d'Albigny à proximité de l'arrivée dans le lac du ruisseau du Colovry dont les eaux présentent encore une qualité biologique douteuse malgré l'existence du collecteur. La forte tolérance aux pollutions biodégradables de Physella (Mouthon 1996), celle de G. laevis étant mal connue, a probablement favorisé son installation dans ce secteur. Dans sa comparaison entre les peuplements de mollusques des lacs du Bourget et d'Annecy, Favre (1940) souligne que $P$. moitessierianum n'a pas encore été observée dans ce dernier. Cependant, aujourd'hui cette espèce, comme $D$. polymorpha et $P$. antipodarum, est très commune dans la zone littorale d'Annecy.

La présence de populations de Sphaeriidae abondantes et bien diversifiées dans la zone littorale du lac témoigne de la bonne qualité biologique de ses habitats à sédiments fins. Cependant, on constate : - depuis les relevés de Favre, la disparition dans la partie nord du lac de Pisidium lilljeborgii et du genre Anodonta que Le Roux (1907-1908) trouvait «en quantité sur la beine nord et parmi les roseaux d'Albigny» ; - qu'à l'exception de $P$. antipodarum, les gastéropodes sont plutôt mal représentés dans la zone littorale du lac. C'est particulièrement le cas pour $V$. piscinalis, $B$. tentacula- ta et différentes espèces de Planorbidae telle que Planorbis carinatus, absente ici, qui sont généralement des éléments dominants de la faune lacustre. En ce qui concerne les Planorbes, Le Roux (1907-1908) mentionne dans son ouvrage que l'on en rencontrait «partout sur le littoral».

La forte réduction de la superficie des ceintures végétales liée aux aménagements de la zone littorale est largement responsable de la disparition ou de la raréfaction de certaines espèces. En revanche, ces aménagements ont sans doute favorisé l'installation récente dans l'histoire du lac d'autres mollusques, et notamment de $D$. polymorpha et de $P$. antipodarum.

L'apparition de $P$. moitessierianum dans le lac d'Annecy est postérieure aux années 40 et, compte tenu de sa sensibilité à la pollution (Mouthon 1996), très probablement à 1964, date de la mise en service du collecteur d'eaux usées de la partie nord du lac. Elle constitue peut-être un des événements les plus marquants de l'histoire malacologique récente de ce milieu. En effet, cette espèce qui vit seulement dans les lacs de faible altitude (Favre 1940, 1941, Walter \& Kuiper 1978, Mouthon 1990) et au niveau du potamon des cours d'eau où elle atteint son préférendum (Meier-Brook 1975, Mouthon 1999) a, contrairement aux autres espèces de Sphaeriidae rencontrées à Annecy, besoin d'une température plus élevée que ces dernières pour se développer. Sa large représentation dans la zone littorale du lac pourrait être une conséquence du récent réchauffement climatique (Houghton et al., 1996). L'observation de ce phénomène sur d'autres plans d'eau, mais également la confrontation de la composition et de la structure des peuplements actuels à celles d'assemblages issus de carottes fossiles réalisées en différents points du lac permettra, sans doute, de confirmer cette hypothèse.

\section{Remerciements}

Cette étude a été réalisée grâce à une subvention du Syndicat du Lac d'Annecy (SILA). Les auteurs remercient M. Christian Perrière responsable du club de plongée "La coulée douce" qui a mis à notre disposition locaux et bateaux ainsi que $M$. Jacques Chappaz qui fut notre pilote avisé pendant toute la campagne d'échantillonnage. Nous remercions également M. Yves Finet (Muséum d'Histoire naturelle de Genève) qui a accepté de nous confier les spécimens de mollusques récoltés par Favre dans la partie nord du lac d'Annecy. 


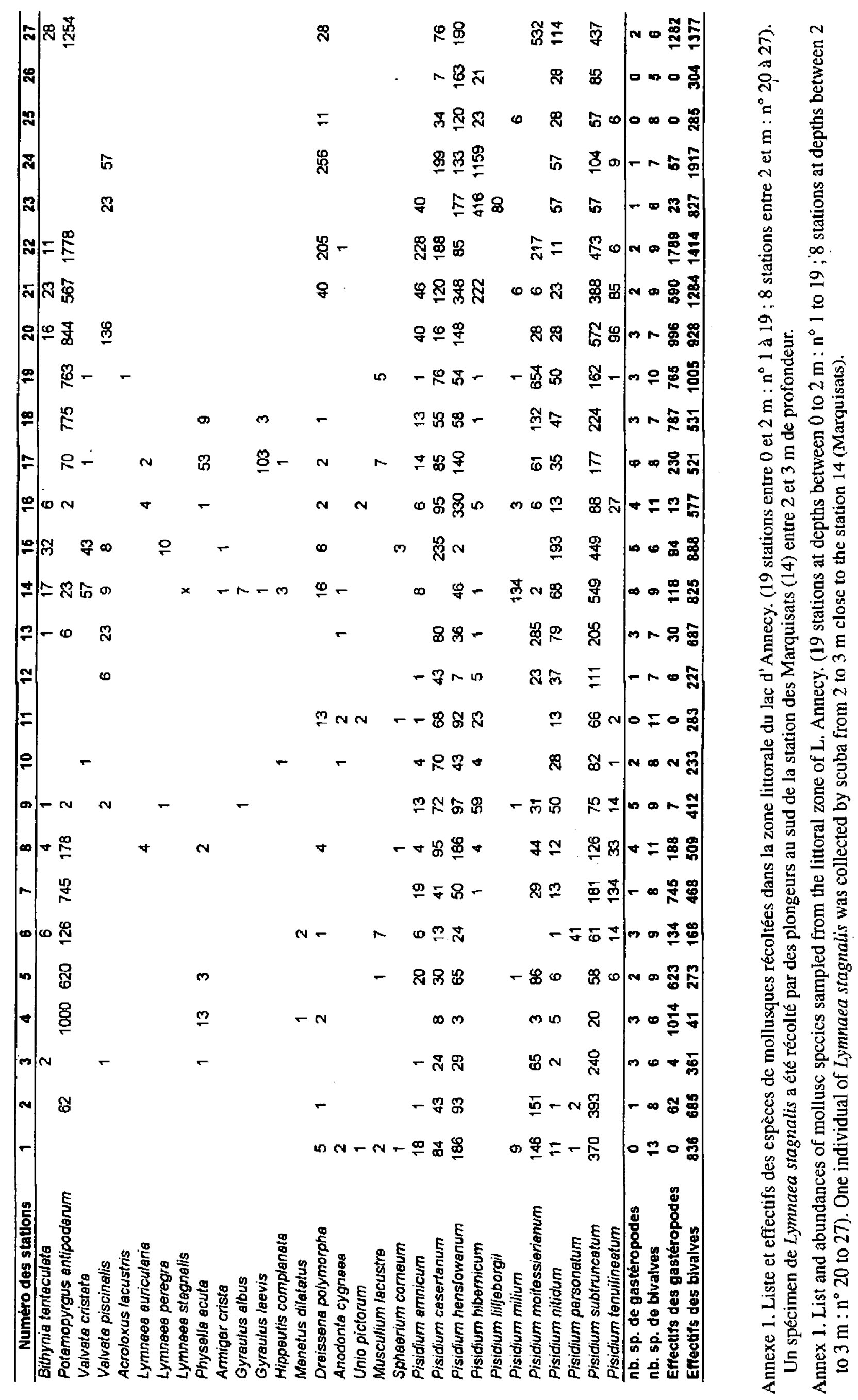




\section{Travaux cités}

Binder E. 1965. - Un mollusque envahissant, la Dreissena polymorpha (Lérnan). Revue mens. Mus., Genève 54 : 2-4.

Crozet B., Pedroli J.C. \& Vaucher C. 1980. - Premières observations de Potamopyrgus jenkinsi (Smith) (Mollusca, Hydrobiidae) en suisse romande. Revue suisse Zool., 87 : 807-811.

Favre J. 1927. - Les mollusques post-glaciaires et actuels du bassin de Genève. Mém. Soc. Phys. et Hist. nat. Genève, 40, $3: 171-434$.

Favre J. 1940. - La faune malacologique post-glaciaire et actuelle du Lac du Bourget. Ann. Ecole nat. Des eaux et Forêts, Paris, 7, $2: 295-444$.

Favre J. 1941, - Les Pisidium du canton de Neuchatel. Soc. Neuch. Sci. Nat., $66: 57-112$.

Juget J. 1967. — La faune benthique du Léman : modalités et déterminismes écologiques du peuplement. Thèse Univ. Lyon : $360 \mathrm{p}$.

Gentile L. de, Picot H., Bourdeau P., Bardet R., Kerjan A., Piriou M., Le Guennic A., Bayssade-Dufour C., Chabasse D. \& Mott K.E. 1996. - La dermatite cercarienne en Europe : un problème de santé publique nouveau ? Bull. O.M.S., 74 : 159-163.

Houghton J.T., Meira Filho L.G., Callender B.A., Harris N., Kattenberg A. \& Maskell K., 1996. - Climate change 1995. The IPCC report. Cambridge University Press : 572 p.

Hubault E. 1947. - Etude thermique, chimique et biologique des eaux des lacs de l'Est de la France. Ann. Ecole nat. des Eaux et Forêts, Paris, 10 (2) : 115-260.

Hubert C. 2000. - La Dermatite cercarienne en France ou 1'occurrence du genre Trichobilharzia en Haute-Savoie (74). Présentation bibliographique et Autécologie d'une population d'hôtes intermédiaires d'une station du lac d'Annecy. DESS, Univ. J. Fourier, Grenoble \& INRA Thonon : $90 \mathrm{p}$.

Kuiper J.G.J. 1966. - La distribution des espèces du genre Pisidium C. PF. en France. J. Conchyl., $105:$ 181-215.
Le Roux M. 1907-1908. - Recherches biologiques sur le lac d'Annecy. Annales de Biologie lacustre, $2: 220-387$.

Martins C. 2000. - Recherches sur l'identification et la pathogénicité de bilharzies (Trematoda, Schistosomatidae) responsables de dermatites humaines en France. DEA Univ. Claude-Bernard, Lyon $1: 40 \mathrm{p}$.

Meier-Brook C. 1975. - Der ökologische Indikatorwert mitteleuropäischer Pisidium Arten (Mollusca, Eulammellibranchiata). Eiszeitaler u. Gegenwart., 26 : 190-195.

Mouthon J. 1981. - Typologie des Mollusques des eaux courantes. Organisation biotypologique et groupements socioécologiques. Ann. Limnol., 17 (2) : 143-162.

Mouthon J. 1986. - Emmericia patula (Gastropoda, Emmericiidae) et Menetus dilatatus (Gastropoda, Planorbidae), deux espèces nouvelles pour la faune de France. Basteria, 50 : 181-188.

Mouthon J. 1990. - Importance des conditions climatiques dans la différenciation des peuplements malacologiques des lacs européens. Arch. Hydrobiol, 118 (3) : 353-370.

Mouthon J. 1996. - Molluscs and biodegradable pollution in rivers : proposal for a scale of sensitivity of species. Hydrobiologia, 317 : 221-229.

Mouthon J. 1999. - Longitudinal organisation of the mollusc species in a theoretical French river. Hydrobiologia, 390:117-128.

Mouthon J.' \& Kuiper J.G.J. 1987. — Inventaire des Sphaeriidae de France. Secrétariat de la Faune et de la Flore et Mus. nat. Hist. nat., (Eds), Paris. Fasc. 41 : 1-60.

Real G. 1974. - Répartition en France de Potamopyrgus jenkinsi (E.A. Smith, 1889). Haliotis, $3: 199-204$.

Walter J.E. \& Kuiper J.G.J. 1978. — Über Verbreitung und Ökologie von Sphaeriiden im Zürichsee (Mollusca : Eulamellibranchiata). Schweiz. Z. Hydrol., 40 (1) : 60-86. 